\title{
COMMUTATIVITY OF RINGS WITH CONSTRAINTS \\ ON NILPOTENTS AND NONNILPOTENTS
}

\author{
MOHAMAD HASANALI \\ Department of Mathematics \\ College of Science and Technology \\ Abudies, Jerusalem, Is rael \\ ADIL YAQUB \\ Department of Mathematics \\ University of California \\ Santa Barbara, California 93106
}

(Received August 27, 1987)

ABSTRACT. Let $R$ be a ring (not necessarily with identity), $N$ the set of nilpotents, and $n>1$ a fixed integer. Suppose that (i) $N$ is commutative; (i1) If $x \neq N$ and $y \notin N$, then $x^{n} y=x y^{n}$; (iii) For $a \varepsilon N$ and $b \varepsilon R$, if $n ![a, b]=0$, then $[a, b]=0$, where $[a, b]=a b$ - ba denotes the commutator. Then $R$ is commutative. This theorem generalizes the $" x^{n}=x^{\prime}$ theorem of Jacobson. It is also shown that above theorem need not be true if any of the hypotheses is deleted, or if " $n$ " in (111) is replaced by "n".

KEY WORDS AND PHRASES. Commutator, nilpotent, Vandermonde determinant. 1980 AMS SUBJECT CLASSIFICATION CODE. 16 A70.

1. INTRODUCTION.

A well known theorem of Jacobson [2] states that a ring $R$ satisfying the identity $\mathrm{x}^{\mathrm{n}}=\mathrm{x}, \mathrm{n}>1$ is fixed, is commutative. Such rings, of course, have no nonzero nilpotents. With this as motivation, we consider the commutativity of a ring satisfying the condition $x^{n} y=x y^{n}$ for all $x \varepsilon R \backslash N, y \varepsilon R \backslash N, n>1$ is fixed, and where $\mathrm{N}$ is assumed to be commutative. That such a ring $\mathrm{R}$ need not be commutative can be seen by taking

$$
R=\left\{\left(\begin{array}{ll}
0 & 0 \\
0 & 0
\end{array}\right),\left(\begin{array}{ll}
0 & 1 \\
0 & 1
\end{array}\right),\left(\begin{array}{ll}
1 & 0 \\
1 & 0
\end{array}\right),\left(\begin{array}{ll}
1 & 1 \\
1 & 1
\end{array}\right) \mid 0,1 \varepsilon \mathrm{GF}(2)\right\}, \mathrm{n}=2
$$


This naturally raises the following question: What extra conditions are needed to guarantee the commutativity of the ground ring $R$ ? Here we give one such extra condition involving commutators. As a corollary of our main theorem, we obtain Jacobson's Theorem (quoted above). We also give examples which show that all the hypotheses of our main theorem are essential.

\section{MAIN RESULTS.}

Our main result may be stated as follows:

MAIN THEOREM. Let $R$ be a ring (not necessarily with identity), $N$ the set of nilpotents, and $n>1$ a fixed integer. Suppose that (1) $N$ is commutative; (11) If $x \notin N$ and $y \notin N$, then $x^{n} y=x y^{n}$; (iii) For $a \varepsilon N$ and $b \varepsilon R, \underline{1 f} n ![a, b]=0$, then $[a, b]=0$. Then $R$ is commutative.

PROOF. Let $x \in R, x \notin N$. Then $x^{2} \notin N$, and hence by (i1), $x^{n}\left(x^{2}\right)=x\left(x^{2}\right)^{n}$, which imp1ies $x^{2 n+1}=x^{n+2}$. Thus,

$$
\left(x-x^{n}\right)^{n+2}=\left(x-x^{n}\right)\left(x-x^{n}\right)^{n+1}=\left(x-x^{n}\right) x^{n+1} g(x)=0,
$$

and hence $x-x^{n} \varepsilon N$ for all $x \notin N$. Since, trivially, this is also true if $x \varepsilon N$, therefore

$$
x-x^{n} \varepsilon N \text { for all } x \in R \text {. }
$$

Next, we prove that

$$
(n !)^{\sigma}[a, b]=0 \text { for some positive integer } \sigma,(a \varepsilon N, b \varepsilon R)
$$

Since $\mathrm{N}$ is commutative, by (i), to prove (2.2) we may assume that $b \notin \mathrm{N}$. Let

$$
u=a+b,(a \varepsilon N, b \notin N) \text {. }
$$

We now distinguish three cases.

CASE 1. ku $\varepsilon N$ for some $k \varepsilon\{1, \ldots, n\}$.

Since $a \varepsilon N$ and $N$ is commutative by (i), therefore (ku)a $=a(k u)$ and hence by (2.3), $k(a+b) a=k a(a+b)$. Thus, $k[a, b]=0$ and hence $n ![a, b]=0$, which proves (2.2) in this case.

CASE 2. $b+k u \in N$ for some $k \in\{1, \ldots, n-1\}$.

Arguing as in Case 1 , we see that $[b+k u, a]=0$. Hence, $[b+k(a+b), a]=0$, which Implies $(k+1)[a, b]=0, k+1 \leqslant n$, and thus $n ![a, b]=0$. Again (2.2) is proved in this case.

CASE 3. $k u \notin N$ for $k=1, \ldots, n$ and $b+k u \notin N$ for $k=1, \ldots, n-1$. 
Recall that $\mathrm{b} \notin \mathrm{N},[$ see $(2.3)]$, and $k u \notin \mathrm{N}$ for $\mathrm{k}=1, \ldots, \mathrm{n}$. Hence by (11),

$$
(k u)^{n} b=(k u) b^{n} \text { for } k=1, \ldots, n
$$

Similarly, since $b \notin N$ and $b+k u \notin N$ for $k=1, \ldots, n-1$, therefore by (1i) again,

$$
(b+k u)^{n} b=(b+k u) b^{n} \text { for } k=1, \ldots, n-1
$$

Setting $k=1$, then $k=2, \ldots$, and finally $k=n-1$ in (2.5), we obtain

$$
\begin{aligned}
& b^{n+1}+A_{1} b+A_{2} b+\ldots+A_{n-1} b+u^{n} b=b^{n+1}+u b^{n} \\
& b^{n+1}+2 A_{1} b+2^{2} A_{2} b+\ldots+2^{n-1} A_{n-1} b+(2 u)^{n} b=b^{n+1}+(2 u) b^{n} \\
& \ldots \ldots \\
& b^{n+1}+(n-1) A_{1} b+(n-1)^{2} A_{2} b+\ldots+(n-1)^{n-1} A_{n-1} b \\
& +((n-1) u)^{n} b=b^{n+1}+((n-1) u) b^{n},
\end{aligned}
$$

where each $A_{1}$ is a sum of terms each of which is a product in which u appears exactly $i$ times and b appears exactly ( $n-1)$ times. Hence, by (2.4) and (2.6), we get

$$
\begin{aligned}
& A_{1} b+A_{2} b+\ldots+A_{n-1} b=0 \\
& 2 A_{1} b+2^{2} A_{2} b+\ldots+2^{n-1} A_{n-1} b=0 \\
& \ldots \\
& (n-1) A_{1} b+(n-1)^{2} A_{2} b+\ldots+(n-1)^{n-1} A_{n-1} b=0
\end{aligned}
$$

The determinant $\Delta$ of the matrix of coefficients of the system of linear equations in $A_{1} b, A_{2} b, \ldots, A_{n-1} b$ in (2.7) is a Vandermonde determinant, and hence $\Delta=$ a product of positive integers each of which is less than $n$. Moreover, it can be seen that $\Delta\left(A_{1} b\right)=0$. A similar argument also shows that $\Delta\left(\mathrm{b}_{1}\right)=0$, and hence $\Delta\left[\mathrm{A}_{1}, \mathrm{~b}\right]=0$. Recalling the definition of $A_{i}$, we see that

$$
A_{1}=u b^{n-1}+b u b^{n-2}+\ldots+b^{n-1} u
$$

and hence

$$
0=\Delta\left[A_{1}, b\right]=\Delta\left[u, b^{n}\right] .
$$


Since $u=a+b,[s e e(2.3)]$, therefore the above equation yields

$$
\Delta\left[a, b^{n}\right]=0,(a \varepsilon N, b \notin N)
$$

Combining (2.9) and (2.1), keeping (1) in mind, we se that

$$
0=\Delta\left[a, b-b^{n}\right]=\Delta[a, b]-\Delta\left[a, b^{n}\right]=\Delta[a, b]
$$

and hence

$$
\Delta[\mathrm{a}, \mathrm{b}]=0,(\mathrm{a} \varepsilon \mathrm{N}, \mathrm{b} \notin \mathrm{N})
$$

Now, combining $(2.10)$ and $(2.8)$, we obtain (taking into account repeated factors of $\Delta)$,

$$
(n !)^{\sigma}[a, b]=0 \text { for some positive integer } \sigma \text {, }
$$

which proves (2.2) in this case also. Thus completes the proof of (2.2).

Returning to the proof of the theorem, note that if $\sigma>1$ then (2.2) implies that

$$
n !\left[a,(n !)^{\sigma-1} b\right]=0
$$

and hence by $(1 i i),\left[a,(n !)^{\sigma-1} b\right]=0$, that is, $(n !)^{\sigma-1}[a, b]=0$. Continuing this process, we eventually obtain $[a, b]=0$ for all a $\varepsilon N, b \neq N$. But, since $N$ is commutative, by (i), therefore,

$$
[a, b]=0 \text { for all a } \varepsilon N, b \in R \text {. }
$$

Combining (2.1) and (2.11), we see that

$$
x-x^{n} \text { is in the center of } R \text {, for all } x \text { in } R \text {, }
$$

and hence $R$ is commutative, by a well known theorem of Herstein [1]. This proves the theorem.

COROLLARY 1. Let $R$ be a ring, $N$ the set of nilpotents, and $n>1$ a fixed integer. Suppose that (i) $N$ is commutative; (ii) If $x \notin N$, then $x^{n}=x$; (iii) For a $\varepsilon N$ and $\mathrm{b} \varepsilon \mathrm{R}, \underline{\text { if }} \mathrm{n} ![\mathrm{a}, \mathrm{b}]=0$, then $[\mathrm{a}, \mathrm{b}]=0$. Then $\mathrm{R}$ is commutative.

As a further corollary, we obtain Jacobson's Theorem [2]:

COROLLARY 2. Let $R$ be a ring and suppose $n>1$ is a fixed integer such that $x^{n}=x$ for all $x$ in $R$. Then $R$ is commutative.

We conclude with the following examples which show that our Main Theorem need not be true if, in hypothesis (iii), " $n$ !" is replaced by " $n$ ", or if any one of the 
hypotheses (i), (ii), (iii) is deleted.

EXAMPLE 1. Let

$$
R=\left\{\left(\begin{array}{ccc}
a & b & c \\
0 & a^{2} & 0 \\
0 & 0 & a
\end{array}\right) \mid a, b, c \in G F(4)\right\} \text {. }
$$

Observe that $R$ satisfies hypothesis ( $i$ ) of our Main Theorem, and also satisfies hypothesis (ii) with $n=7$. But hypothesis (iii) is not satisfied for this value of $n$. However, if $n$ ! is replaced by $n$ in hypothesis (iii), then $R$ would satisfy this new hypothesis, $(n=7)$. This example shows that " $n$ !" cannot be replaced by "n" in (iii).

EXAMPLE 2. Let

$$
R=\left\{\left(\begin{array}{ccc}
0 & a & b \\
0 & 0 & c \\
0 & 0 & 0
\end{array}\right) \mid a, b, c \in G F(3)\right\}, n=2
$$

It is easily checked that $R$ satisfies all the hypotheses of our Main Theorem except hypothesis ( $i$ ), but $R$ is not commutative. Hence, ( $i$ ) cannot be deleted.

EXAMPLE 3. Let $R$ be the ring of quaternions, and let $n>1$ be any positive integer. Note that $R$ satisfies all the hypotheses of our Main Theorem except hypothesis ( $i i)$. Hence, (ii) cannot be deleted.

EXAMPLE 4. Let

$$
R=\left\{\left(\begin{array}{ll}
0 & 0 \\
0 & 0
\end{array}\right),\left(\begin{array}{ll}
1 & 1 \\
1 & 1
\end{array}\right),\left(\begin{array}{ll}
0 & 1 \\
0 & 1
\end{array}\right),\left(\begin{array}{ll}
1 & 0 \\
1 & 0
\end{array}\right) \mid 0,1 \varepsilon G F(2)\right\}, n=2
$$

It is readily verified that all the hypotheses of our Main Theorem are satisfied except hypothesis (iii). Hence, (iii) cannot be deleted, since $R$ is not commutative.

\section{REFERENCES}

1. HERSTEIN, I., A generalization of a theorem of Jacobson, I., Amer. J. Math, 73 (1951), 755-762.

2. JACOBSON, N., Structure of rings, A.M.S. Collog. Pub1. (1964). 


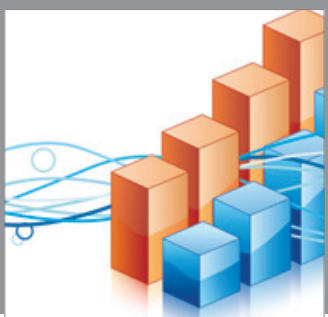

Advances in

Operations Research

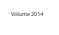

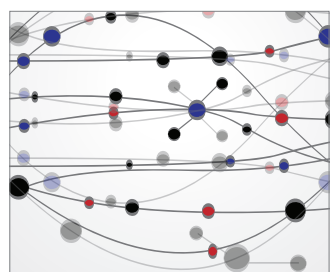

\section{The Scientific} World Journal
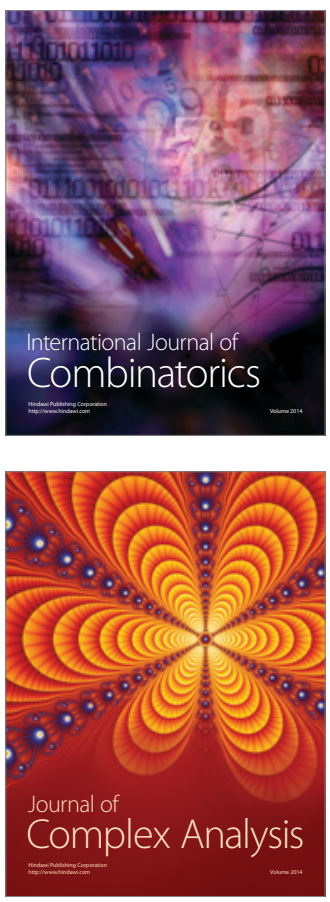

International Journal of

Mathematics and

Mathematical

Sciences
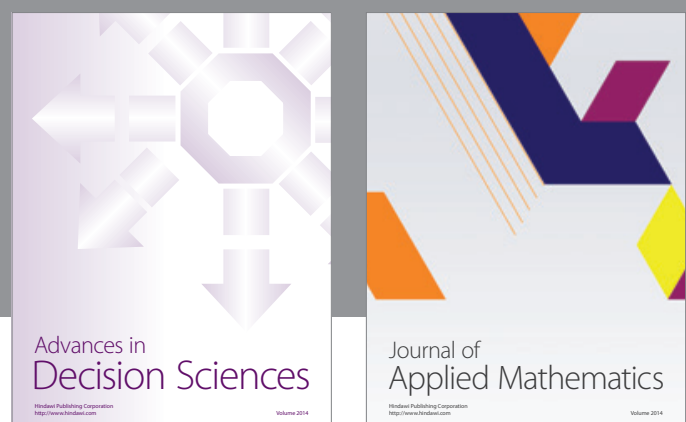

Journal of

Applied Mathematics
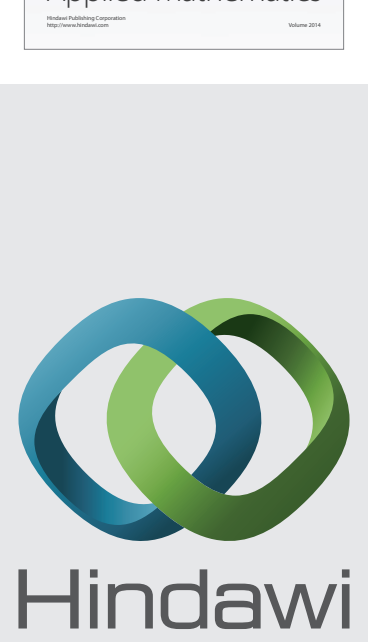

Submit your manuscripts at http://www.hindawi.com
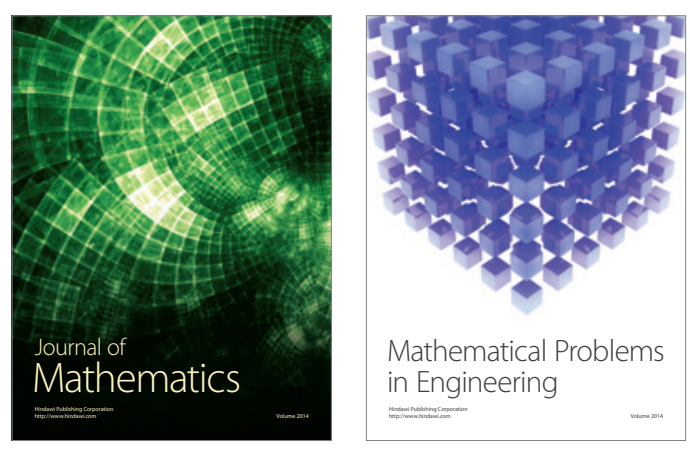

Mathematical Problems in Engineering
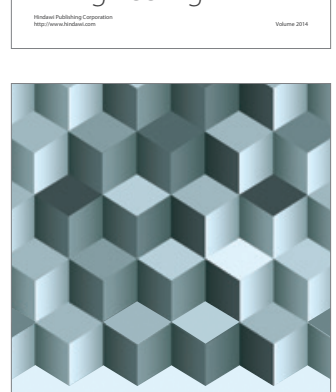

Journal of

Function Spaces
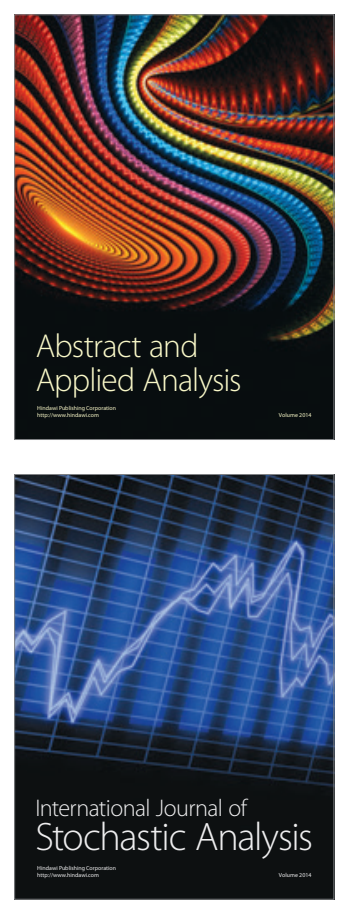

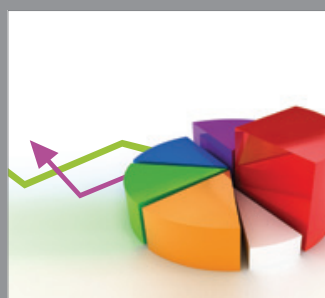

ournal of

Probability and Statistics

Promensencen
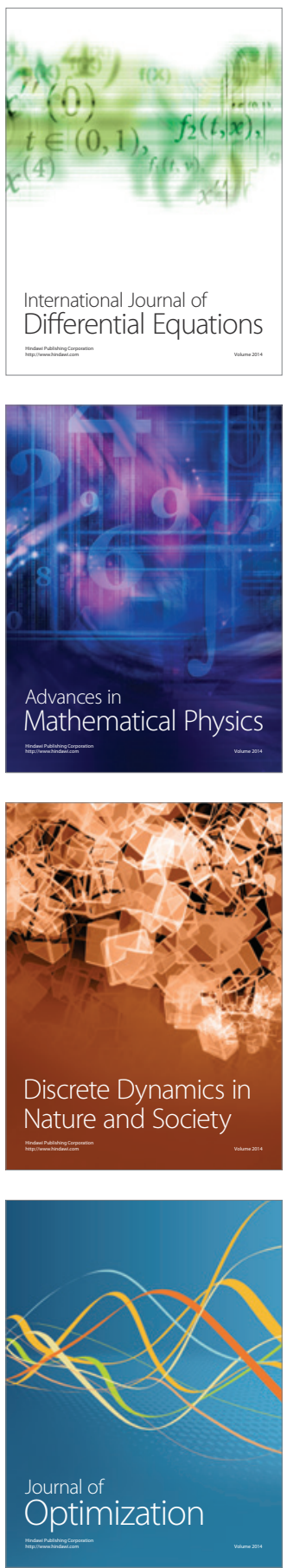